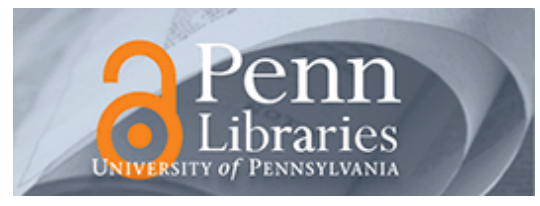

University of Pennsylvania

ScholarlyCommons

Statistics Papers

Wharton Faculty Research

$7-2000$

\title{
Percolation in a Dependent Random Environment
}

Johan Jonasson

Elchanan Mossel

University of Pennsylvania

Yuval Peres

Follow this and additional works at: https://repository.upenn.edu/statistics_papers

Part of the Statistics and Probability Commons

\section{Recommended Citation}

Jonasson, J., Mossel, E., \& Peres, Y. (2000). Percolation in a Dependent Random Environment. Random Structures \& Algorithms, 16 (4), 333-343. http://dx.doi.org/10.1002/1098-2418(200007)16:4<333::AIDRSA3>3.0.CO;2-C

This paper is posted at ScholarlyCommons. https://repository.upenn.edu/statistics_papers/434

For more information, please contact repository@pobox.upenn.edu. 


\title{
Percolation in a Dependent Random Environment
}

\author{
Abstract \\ Draw planes in $\mathbb{R}^{3}$ that are orthogonal to the $x$ axis, and intersect the $x$ axis at the points of a Poisson \\ process with intensity $\lambda$; similarly, draw planes orthogonal to the $y$ and $z$ axes using independent Poisson \\ processes (with the same intensity). Taken together, these planes naturally define a randomly stretched \\ rectangular lattice. Consider bond percolation on this lattice where each edge of length is open with \\ probability $e^{-}$, and these events are independent given the edge lengths. We show that this model exhibits \\ a phase transition: for large enough $\lambda$ there is an infinite open cluster a.s., and for small $\lambda$ all open \\ clusters are finite a.s. We prove this result using the method of paths with exponential intersection tails, \\ which is not applicable in two dimensions. The question whether the analogous process in the plane \\ exhibits a phase transition is open. \\ Disciplines \\ Statistics and Probability
}




\title{
Percolation in a Dependent Random Environment
}

\author{
Johan Jonasson $\quad$ Elchanan Mossel $\quad$ Yuval Peres \\ Chalmers University of Technology and Hebrew University of Jerusalem
}

February 8, 2000

\begin{abstract}
Draw planes in $\mathbb{R}^{3}$ that are orthogonal to the $x$ axis, and intersect the $x$ axis at the points of a Poisson process with intensity $\lambda$; similarly, draw planes orthogonal to the $y$ and $z$ axes using independent Poisson processes (with the same intensity). Taken together, these planes naturally define a randomly stretched rectangular lattice. Consider bond percolation on this lattice where each edge of length $\ell$ is open with probability $e^{-\ell}$, and these events are independent given the edge lengths. We show that this model exhibits a phase transition: for large enough $\lambda$ there is an infinite open cluster a.s., and for small $\lambda$ all open clusters are finite a.s. We prove this result using the method of paths with exponential intersection tails, which is not applicable in two dimensions. The question whether the analogous process in the plane exhibits a phase transition is open.
\end{abstract}

\section{Introduction}

Let $G=(V, E)$ be a graph, and suppose that the edges are labeled with (possibly dependent) random variables $\left\{X_{e}\right\}_{e \in E}$, taking values in $[0,1]$. We call the collection $\left\{X_{e}\right\}_{e \in E}$ the environment, and consider independent percolation, $\left\{\eta_{e}\right\}_{e \in E}$, in the random environment $\left\{X_{e}\right\}$. More precisely we do the following:

- Take a sample of the variables $\left\{X_{e}\right\}$ according to their joint distribution.

- Set each edge open (i.e., set $\eta_{e}=1$ ) with probability $X_{e}$ and closed (i.e., $\eta_{e}=0$ ) otherwise, independently for all edges.

Theorem 1.1 Let $G=\mathbf{Z}^{d}$ for $d \geq 3$. There exists a number $\theta(d)<1$ such that if an environment $\left\{X_{e}\right\}_{e \in \mathbf{Z}^{d}}$ satisfies

(A) $\sup _{e \in E} \mathbf{E}\left[X_{e}^{-1}\right]<\theta(d)^{-1}$;

(B) For any oriented path $\varphi$ in $\mathbf{Z}^{d}$ and any collection of $n$ distinct edges $\left\{e_{j}\right\}_{j=1}^{n}$ on $\varphi$, the corresponding variables $\left\{X_{e_{j}}\right\}_{j=1}^{n}$ are independent.

then a.s., there exists an infinite oriented percolation cluster which is transient for simple random walk.

(The oriented cluster of a vertex $v$ is the union of all open oriented paths emanating from $v$.) 
Corollary 1.2 Given $0<\lambda<\infty$, consider a random environment satisfying the independence condition (B) of the theorem, such that $\mathbf{P}\left[X_{e} \leq x\right]=x^{\lambda}$ for $x \in[0,1]$ and $e \in E$. If $d \geq 3$ and $\lambda>(1-\theta(d))^{-1}$, then a.s. there exists a transient oriented percolation cluster.

Proof: $\mathbf{E}\left[X_{e}^{-1}\right]=\int_{0}^{1} t^{-1} \lambda t^{\lambda-1} d t=\lambda /(\lambda-1)$, so condition (A) holds provided that $\lambda>(1-\theta(d))^{-1}$.

Consider $d$ independent Poisson point processes with parameter $\lambda$. The $d$-fold Cartesian product of these point processes, with the standard product adjacency relation, is a graph isomorphic to $\mathbf{Z}^{d}$ that we refer to as a "stretched lattice". Set an edge $e$ of length $L_{e}$ open with probability $X_{e}=\exp \left(-L_{e}\right)$, independently for all edges given their lengths. Since $\mathbf{P}\left[X_{e} \leq x\right]=\mathbf{P}\left[L_{e} \geq \log (1 / x)\right]=x^{\lambda}$, Corollary 1.2 applies to this process. In Section 3 we prove Proposition 3.1, which together with Corollary 1.2, establishes that this percolation process has a phase-transition in $\lambda$. It is not known if such a phase transition occurs for $d=2$ (see Conjecture 3.2). See figure 1 below for a picture of this process in 2 dimensions. This picture was produced using software written by A. Scolnicov.

Figure 1: Percolation on the 2-dimensional stretched lattice with $\lambda=2.5$

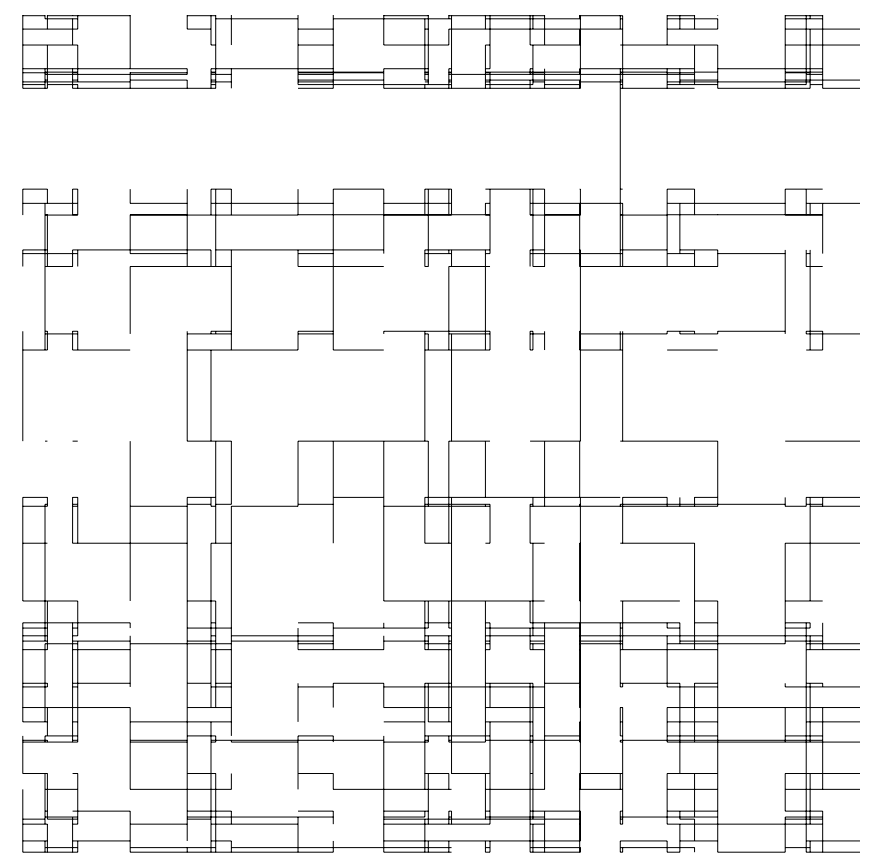

The rest of the paper is organized as follows. Theorem 1.1 is proved in Section 2 via the method of paths with Exponential Intersection Tails (EIT). This method was introduced in Cox and Durrett [5] as a tool for bounding critical probabilities, and developed further by Benjamini, Pemantle and Peres in [3], who used it to prove transience of oriented clusters for i.i.d. percolation.

In Section 3 we analyze the "stretched lattice" mentioned above, and in Section 4 we discuss another application of the theorem to prove the existence of a phase transition. 


\section{Proofs}

\section{Definition 2.1 [3]:}

- For a graph $G=(V, E)$ and a vertex $v_{0} \in V$, we denote by $\Upsilon\left(G, v_{0}\right)$ the collection of infinite paths $\varphi$ which emanate from $v_{0}$, such that the $n^{\text {th }}$ vertex on $\varphi$ is at distance $n$ from $v_{0}$ (in the graph metric).

- Let $0<\theta<1$. A Borel probability measure $\mu$ on $\Upsilon\left(G, v_{0}\right)$ has Exponential intersection tails with parameter $\theta$ (in short, $E I T(\theta)$ ) if there exists $C$ such that

$$
\mu \times \mu\{(\varphi, \psi):|\varphi \cap \psi| \geq n\} \leq C \theta^{n},
$$

where $|\varphi \cap \psi|$ is the number of edges common to $\varphi$ and $\psi$.

The proof of Theorem 1.1 uses the following lemma.

Lemma $2.2[\mathbf{5}, \mathbf{3}]$ : For all $d \geq 3$ there exists a $\theta=\theta(d)<1$, and a measure $\mu$ supported on oriented paths emanating from 0 , such that $\mu$ has $\operatorname{EIT}(\theta)$.

For $d \geq 4$ one can take the uniform measure on oriented paths (see Cox and Durrett $[5])$. Moreover, using this measure they have the following asymptotic bounds for $\theta(d)$ :

Proposition $2.3[5]: \quad \theta(d) \leq d^{-1}+d^{-3}+O\left(d^{-4}\right) \quad$ as $d \rightarrow \infty$.

For $d=3$ the construction of a measure with EIT is more difficult (see [3] and [10]). We note that there is no measure with the EIT property in dimension $d=2$, so the methods of this paper are restricted to dimensions $d>2$.

We will prove a generalization of Theorem 1.1. Denote by $\mathbf{P}_{X}$ the (product) probability measure given the variables $\left\{X_{e}\right\}$ and by $\mathbf{P}$ the unconditional probability measure. Let $\mathbf{E}_{X}$ and $\mathbf{E}$ denote the corresponding expectation operators. For a subset $\Gamma$ of $\Upsilon\left(G, v_{0}\right)$, denote by $\mathcal{C}\left(v_{0}, \Gamma\right)$ the union of all open paths that are prefixes of paths in $\Gamma$. We consider $\mathcal{C}\left(v_{0}, \Gamma\right)$ as an undirected graph, and say that $\mathcal{C}\left(v_{0}, \Gamma\right)$ is transient, if simple random walk on $\mathcal{C}\left(v_{0}, \Gamma\right)$ is transient.

Theorem 2.4 Let $G=(V, E)$ be a graph. Suppose that $\mu$ is a probability measure supported on $\Gamma \subset \Upsilon\left(G, v_{0}\right)$, such that $\mu$ has $\operatorname{EIT}(\theta)$. Let $X=\left\{X_{e}\right\}_{e \in E}$ be an environment that satisfies:

(A) $r:=\sup _{e \in E} \mathbf{E}\left[X_{e}^{-1}\right]<\theta^{-1} ;$

(B) If $\left\{e_{i}\right\}_{i=1}^{n}$ are distinct edges of a path $\varphi \in \Gamma$, then $\left\{X_{e_{i}}\right\}_{i=1}^{n}$ are independent.

Then $\mathbf{P}_{X}\left[\mathcal{C}\left(v_{0}, \Gamma\right)\right.$ is transient $]>0$ for a.e. $X$.

Proof of Theorem 1.1: For each $d \geq 3$, fix $\theta(d)$ as in Lemma 2.2. Denote by $T_{o r}$ the event that there exists a transient oriented cluster in $\mathbf{Z}^{d}$. By Theorem 2.4, $\mathbf{P}_{X}\left(T_{o r}\right)>0$ for a.e. environment $X$. Since $T_{o r}$ is a tail event for the sequence of random variables $\left\{\eta_{e}\right\}_{e \in E}$, and these variables are conditionally independent given $X$, Kolmogorov's zeroone law implies that $\mathbf{P}_{X}\left(T_{o r}\right)=1$ for a.e. environment $X$.

Proof of Theorem 2.4: We first show that for a.e. environment $X$,

$$
\mathbf{P}_{X}\left[\mathcal{C}\left(v_{0}, \Gamma\right) \text { is infinite }\right]>0 .
$$


For a path $\varphi$ and $n \geq 1$, we let $\varphi_{n}$ be the prefix of $\varphi$ of length $n$. Let

$$
Z_{n}=\int_{\Gamma} \prod_{e \in \varphi_{n}} X_{e}^{-1} O_{\varphi_{n}} d \mu(\varphi)
$$

where $O_{\varphi_{n}}$ is the indicator of the event that $\varphi_{n}$ is open in the percolation.

Let $\mathcal{F}_{n}$ denote the $\sigma$-algebra generated by the environment $X$ and the status $\eta_{e}$ of edges $e=(v, w)$ with $d\left(v_{0}, w\right) \leq n$ and $d\left(v_{0}, v\right) \leq n$. If $e_{i}(\varphi)$ denotes the $i$ 'th edge of $\varphi$, then by Fubini's theorem:

$$
\mathbf{E}_{X}\left[Z_{n} \mid \mathcal{F}_{n-1}\right]=\mathbf{E}_{X}\left[\int_{\Gamma} \prod_{i=1}^{n} X_{e_{i}(\varphi)}^{-1} O_{\varphi_{n}} d \mu(\varphi) \mid \mathcal{F}_{n-1}\right]=\int_{\Gamma} \prod_{i=1}^{n-1} X_{e_{i}(\varphi)}^{-1} O_{\varphi_{n-1}} d \mu(\varphi)=Z_{n-1}
$$

Thus $Z_{n}$ is a $\mathbf{P}_{X}$-martingale with respect to the filtration $\mathcal{F}_{n}$ for each environment $X$. Denote

$$
Z_{\infty}:=\limsup _{n \rightarrow \infty} Z_{n}
$$

Since $Z_{n}$ is non-negative, it converges $\mathbf{P}_{X}$-a.s. to $Z_{\infty}$. We note that if $Z_{\infty}>0$, then $\mathcal{C}\left(v_{0}, \Gamma\right)$ is infinite. By definition, $\mathbf{E}_{X}\left[Z_{n}\right]=1$. Thus in order to prove (1), it is enough to show that for a.e. $X$, the sequence $Z_{n}$ converges to $Z_{\infty}$ in $L^{2}\left(\mathbf{P}_{X}\right)$ (since convergence in $L^{2}$ implies convergence in $L^{1}$, so $\mathbf{E}_{X}\left[Z_{\infty}\right]=1$ and $\mathbf{P}_{X}\left[Z_{\infty}>0\right]>0$ ).

By Fubini's Theorem we have for all $X$ :

$$
\begin{aligned}
\mathbf{E}_{X}\left[Z_{n}^{2}\right] & =\mathbf{E}_{X}\left[\int_{\Gamma \times \Gamma} \prod_{e \in \varphi_{n}} X_{e}^{-1} \prod_{e \in \psi_{n}} X_{e}^{-1} O_{\varphi_{n}} O_{\psi_{n}} d \mu(\varphi) d \mu(\psi)\right] \\
& =\int_{\Gamma \times \Gamma} \prod_{e \in \varphi_{n} \cap \psi_{n}} X_{e}^{-1} d \mu(\varphi) d \mu(\psi) .
\end{aligned}
$$

By condition (B) and another application of Fubini's Theorem,

$$
\mathbf{E}\left[Z_{n}^{2}\right]=\mathbf{E}\left[\mathbf{E}_{X}\left[Z_{n}^{2}\right]\right]=\int_{\Gamma \times \Gamma} \prod_{e \in \varphi_{n} \cap \psi_{n}} \mathbf{E}\left[X_{e}^{-1}\right] d \mu(\varphi) d \mu(\psi) .
$$

By the $\operatorname{EIT}(\theta)$ property and condition (A), the last integral is at most

$$
C \sum_{m=1}^{\infty} r^{m} \theta^{m}=C_{1}<\infty
$$

The sequence $\mathbf{E}_{X}\left(Z_{n}^{2}\right)$ is monotone increasing for any $X$, so by the monotone convergence theorem

$$
\mathbf{E}\left[\lim _{n} \mathbf{E}_{X}\left(Z_{n}^{2}\right)\right]=\lim _{n} \mathbf{E}\left[Z_{n}^{2}\right] \leq C_{1} .
$$

Thus, for a.e. $X$ the martingale $\left\{Z_{n}\right\}$ is uniformly bounded in $L^{2}\left(\mathbf{P}_{X}\right)$. (1) follows.

Next, we study transience of $\mathcal{C}\left(v_{0}, \Gamma\right)$. In order to prove the transience of a graph, it suffices to show that the graph supports a flow (i.e., a real-valued antisymmetric function defined on the set of directed edges such that the net flow into any vertex 
except the source is zero) with finite energy (see Doyle and Snell [6]). For each $N \geq 1$ and for every directed edge $e^{\prime}=(v, w) \in E$ such that $d\left(v_{0}, v\right)<d\left(v_{0}, w\right)$, we define

$$
f_{N}(v, w)=f_{N}\left(e^{\prime}\right)=\int_{\Gamma} \prod_{e \in \varphi_{N}} X_{e}^{-1} O_{\varphi_{N}} 1_{\left\{e^{\prime} \in \varphi_{N}\right\}} d \mu(\varphi)
$$

and set $f_{N}(w, v)=-f_{N}(v, w)$,

We note that $f_{N}$ is a flow on the edges of $\mathcal{C}\left(v_{0}, \Gamma\right)$ from $v_{0}$ to $\left\{v: d\left(v_{0}, v\right)>N\right\}$. The strength of $f_{N}$ (the total outflow from $v_{0}$ ) is exactly $Z_{N}$. We now estimate the expected energy of $f_{N}$ :

$$
\begin{aligned}
& \mathbf{E}_{X}\left[\sum_{e^{\prime}} f_{N}\left(e^{\prime}\right)^{2}\right] \\
& =\mathbf{E}_{X}\left[\int_{\Gamma \times \Gamma} \prod_{e \in \varphi_{N}} X_{e}^{-1} \prod_{e \in \psi_{N}} X_{e}^{-1} O_{\varphi_{N}} O_{\psi_{N}} \sum_{e^{\prime}} \mathbf{1}_{\left\{e^{\prime} \in \varphi_{N}, e^{\prime} \in \psi_{N}\right\}} d \mu(\varphi) d \mu(\psi)\right] \\
& =\int_{\Gamma \times \Gamma}\left|\varphi_{N} \cap \psi_{N}\right| \prod_{e \in \varphi_{N} \cap \psi_{N}} X_{e}^{-1} d \mu(\varphi) d \mu(\psi) .
\end{aligned}
$$

Therefore,

$$
\mathbf{E}\left[\sum_{e^{\prime}} f_{N}\left(e^{\prime}\right)^{2}\right]=\int_{\Gamma \times \Gamma}\left|\varphi_{N} \cap \psi_{N}\right| \prod_{e \in \varphi_{N} \cap \psi_{N}} \mathbf{E}\left[X_{e}^{-1}\right] d \mu(\varphi) d \mu(\psi) .
$$

By the $\operatorname{EIT}(\theta)$ property and condition (A), the last integral is bounded by:

$$
C \sum_{n=0}^{\infty} n \theta^{n} r^{n}<\infty
$$

It is easy to see that for every $X$ and for each edge $e$, the sequence $\left\{f_{n}(e)\right\}$ is a (nonnegative) martingale adapted to $\mathcal{F}_{n}$. Then for every $X$, the limiting edge-function $f(e):=\lim f_{n}(e)$ defines a flow of strength $Z_{\infty}$. By equation (5) and Fatou's Lemma, this flow has finite energy for a.e. $X$. This implies that for a.e. $X$,

$$
\mathbf{P}_{X}\left[\mathcal{C}\left(v_{0}, \Gamma\right) \text { is transient }\right] \geq \mathbf{P}_{X}\left[Z_{\infty}>0\right]>0 .
$$

\section{Percolation on a randomly stretched lattice}

Denote by $E^{\prime}$ the collection of edges on the axes in $\mathbf{Z}^{d}$. Let $\left\{L_{e^{\prime}}\right\}_{e^{\prime} \in E^{\prime}}$ be i.i.d. exponential random variables with parameter $\lambda$. If $e$ is any edge in $\mathbf{Z}^{d}$ that is parallel to $e^{\prime} \in E^{\prime}$ (i.e., the projection of $e$ on some axis gives $e^{\prime}$ ) then set $L_{e}=L_{e^{\prime}}$ and $X_{e}=\exp \left(-L_{e}\right)$. The discussion following Corollary 1.2 shows that this model is covered by that corollary, and justifies the terminology "stretched lattice".

It is easy to see that this model is monotone in $\lambda$ : As $\lambda$ increases, the probability that there exists a (oriented/transient) percolation cluster increases. 


\subsection{Percolation}

The existence of a phase transition for this model follows from Corollary 1.2 together with the following proposition, which proves that when $\lambda$ is small percolation is impossible. This was first established by Olle Häggström (private communication) for $\mathbf{Z}^{2}$. For completeness, we reproduce (and generalize) the argument.

Proposition 3.1 For all $d \geq 2$, if

$$
\lambda \leq \frac{1}{d-1}
$$

then almost surely, all open clusters are finite.

Proof: We use a Borel-Cantelli argument to show that whenever (6) holds, the open cluster containing 0 is finite a.s. Let $A_{n}$ be the event that there exists a box containing the origin, with all its faces in the region $\left\{x: 2^{n}<|x|_{\infty} \leq 2^{n+1}\right\}$, such that all edges connecting the box to its complement are closed. Clearly, on $\cup_{n} A_{n}$, the origin is not in an infinite cluster. Moreover, the events $A_{n}$ are independent. For $2^{n}<|s| \leq 2^{n+1}$, we let $A_{s}^{i,+}$ be the event that all edges $e=\left(v, v+\xi_{i}\right)$ for $|v|_{\infty} \leq 2^{n+1}$ with $v_{i}=s$, are closed (Here $\left\{\xi_{i}\right\}_{i=1}^{d}$ are the standard unit vectors in $\mathbf{Z}^{d}$ ). Similarly we define $A_{s}^{i,-}$. We have

$$
\begin{aligned}
\mathbf{P}\left[A_{n}\right] & \geq \mathbf{P}\left[\cap_{i=1}^{d}\left(\cup_{2^{n}<s \leq 2^{n+1}} A_{s}^{i,+}\right) \cap\left(\cap_{i=1}^{d}\left(\cup_{-2^{n+1} \leq s<-2^{n}} A_{s}^{i,-}\right)\right)\right] \\
& =\mathbf{P}\left[\left(\cup_{2^{n}<s \leq 2^{n+1}} A_{s}^{1,+}\right)\right]^{2 d}
\end{aligned}
$$

For each $s$, let $X_{s}$ be the environment variable which corresponds to edges $e=\left(v, v+\xi_{1}\right)$ with $v_{1}=s$. On one hand:

$$
\mathbf{P}\left[\min _{2^{n}<s \leq 2^{n+1}} X_{s}<2^{-n / \lambda}\right]=1-\left(1-\left(2^{-n / \lambda}\right)^{\lambda}\right)^{2^{n}} \geq 1-e^{-1},
$$

and on the other hand for $2^{n}<t \leq 2^{n+1}$,

$$
\mathbf{P}\left[\cup_{2^{n}<s \leq 2^{n+1}} A_{s}^{1,+} \mid X_{t}<2^{-n / \lambda}\right] \geq \mathbf{P}\left[A_{t}^{1,+} \mid X_{t}<2^{-n / \lambda}\right] \geq\left(1-2^{-n / \lambda}\right)^{\left(\left(2^{n+2}+1\right)^{d-1}\right)} .
$$

Combining (8) and (9) with (7) we obtain:

$$
\mathbf{P}\left[A_{n}\right] \geq\left(\left(1-e^{-1}\right)\left(1-2^{-n / \lambda}\right)^{\left(\left(2^{n+2}+1\right)^{d-1}\right)}\right)^{2 d}
$$

which is bounded away from zero once (6) holds. It now follows from the Borel-Cantelli Lemma that (6) implies $\mathbf{P}\left[A_{n}\right.$ i.o $]=1$.

Remark. Since $\mathbf{Z}^{2}$ does not support a measure with the EIT property, the technique used to prove Corollary 1.2 does not work for $d=2$. However we believe, but do not know how to prove:

Conjecture 3.2 If $d=2$, then there exists $\lambda_{c} \geq 1$ such that for $\lambda>\lambda_{c}$ there a.s. exists an infinite percolation cluster (perhaps even $\lambda_{c}=1$ ). 
It is worth noting that we also have the following variant of Proposition 3.1. As usual, we say that the distribution of a random variable $L$ is heavy-tailed if

$$
\lim _{x \rightarrow \infty} \frac{\log P[L>x]}{x}=0 .
$$

Proposition 3.3 If the random variables $L_{e^{\prime}}$ for $e^{\prime} \in E^{\prime}$, are i.i.d. with some heavytailed distribution (with the rest of the model unchanged), then there are a.s. no infinite percolation clusters.

The proof is analogous to that of Proposition 3.1. Indeed with $A_{n}$ defined as in that proof, we have $P\left[A_{n}\right] \rightarrow 1$.

A consequence of Proposition 3.3 is that when the lengths of the edges are heavytailed, we can take the marginal probability for an edge to be open in the percolation arbitrarily close to 1 , but still have no infinite components.

\subsection{Dual percolation}

Define as usual the dual lattice $\mathbf{Z}_{*}^{2}$ by setting $\mathbf{Z}_{*}^{2}=\left(V^{*}, E^{*}\right)$ where $V^{*}$ is the set of faces (i.e. the set of squares of area 1 ) of $\mathbf{Z}^{2}$ and $\left(u^{*}, v^{*}\right) \in E^{*}$ iff $u^{*}$ and $v^{*}$ have a side (i.e. and edge $e \in E$ ) in common. The dual percolation model is defined by setting $\eta_{e^{*}}^{*}=1$ iff $\eta_{e}=0$ for the edge $e \in E$ crossing $e^{*}$.

Proposition 3.4 Let $\lambda<1$. Then with probability 1 there exists an infinite dual percolation cluster.

Proof: Fix some vertex $0^{*} \in V^{*}$ and call it the origin of $\mathbf{Z}_{*}^{2}$. Denote by $B_{n \times m}^{*}$ the $2 n \times 2 m$-box centered at $0^{*}$, i.e. the subgraph of $Z_{*}^{2}$ induced by the vertices $\left\{u^{*}:\left|u_{1}^{*}\right| \leq\right.$ $\left.n,\left|u_{2}^{*}\right| \leq m\right\}$. Define the events $E_{1}, E_{2}, \ldots$ by letting $E_{2 k-1}$ be the event that there exists a path of dually open edges connecting the north side and the south side of the box $B_{2^{2 k-1} \times 2^{2 k}}^{*}$ and by letting $E_{2 k}$ be the event that there exists a path of dually open edges connecting the west side and the east side of the box $B_{2^{2 k+1} \times 2^{2 k}}^{*}$. Then for obvious geometrical reasons we have that if $\mathbf{P}\left[E_{n}^{c}\right.$ i.o. $]=0$, then an infinite dual percolation cluster exists with probability 1.

We shall prove that all but finitely many boxes will in fact be crossed by some straight line of dually open edges: Assume that $n$ is even. Then $E_{n} \supseteq \cup_{i=1}^{2^{n+1}} A_{i}$ where $A_{i}$ is the event that the path $\left(-2^{n+1},-2^{n}+i\right)\left(-2^{n+1}+1,-2^{n}+i\right) \ldots\left(2^{n+1},-2^{n}+i\right)$ is dually open. Now the dependence in the environment translated to the dual model is such that $A_{i}$ 's are mutually independent whereas the edges along the path defining one $A_{i}$ are heavily dependent. Thus $\mathbf{P}\left[E_{n}^{c}\right] \leq \mathbf{P}\left[A_{1}^{c}\right]^{2^{n+1}}$. By conditioning on the length of the edges $e \in E$ crossing the path defining $A_{1}$, we have (substituting $t=e^{-\lambda l}$ ):

$$
\begin{aligned}
\mathbf{P}\left[A_{1}\right] & =\lambda \int_{0}^{\infty}\left(1-e^{-l}\right)^{2^{n+2}} e^{-\lambda l} d l \\
& =\int_{0}^{1}\left(1-t^{\lambda^{-1}}\right)^{2^{n+2}} d t \geq \int_{0}^{2^{-\lambda(n+1)}}\left(1-2^{-(n+1)}\right)^{2^{n+2}} d t \geq K 2^{-\lambda(n+1)}
\end{aligned}
$$

for some nonzero constant $K$ which is independent of $n$. Thus

$$
\mathbf{P}\left[E_{n}^{c}\right] \leq \mathbf{P}\left[A_{1}^{c}\right]^{2^{n+1}} \leq\left(1-K 2^{-\lambda(n+1)}\right)^{2^{n+1}} \leq e^{-K 2^{(1-\lambda)(n+1)}}
$$

Also, by rotation invariance, this bound holds for odd $n$ as well. Therefore $\sum_{n=1}^{\infty} \mathbf{P}\left[E_{n}^{c}\right]<$ $\infty$ and it follows from the Borel-Cantelli Lemma that $\mathbf{P}\left[E_{n}^{c}\right.$ i.o. $]=0$.

In analogy with the above we also have a variant for heavy-tailed distributions: 
Proposition 3.5 If the $L_{e^{\prime}} ' s, e^{\prime} \in E^{\prime}$, are i.i.d. with some heavy-tailed distribution (with the rest of the model unchanged) then with probability 1 there exists a dual infinite cluster.

The dual percolation result has an application to the following probability model for patterns of black and white polygons: Fix a (large) box $B$ in $\mathbb{R}^{2}$ and generate infinite vertical and horizontal lines according to two independent Poisson processes of intensity $\lambda$ as we did when we constructed the stretched lattice. Then the intersection of $B$ with the resulting lines produces a pattern of rectangles in $B$. Now suppose $\mathbb{R}^{2} \backslash B$ is colored with one of the two colors and let us, given the pattern of rectangles, color the rectangles in $B$ black or white randomly in such a way that each outcome $x$ is given a probability, $\mu_{B}(x)$, given by

$$
\mu_{B}(x)=\frac{1}{Z} e^{-2 l(x)}
$$

where $Z$ is a normalizing constant (which depends on the pattern of rectangles) and $l(x)$ is the total length of the boundary between the black and white regions of $\mathbb{R}^{2}$.

Now the rectangles of the basic stretched lattice are the vertices of the dual $\mathbf{Z}_{*}^{2}$, so the measure $\mu_{B}$ is a Gibbs measure for the Ising model with a one-spin boundary condition where the interaction $J_{\left(u^{*}, v^{*}\right)},\left(u^{*}, v^{*}\right) \in E^{*}$, is given by the length of the edge $e \in E$ crossing $\left(u^{*}, v^{*}\right)$. Letting $B \uparrow \mathbb{R}^{2}$, the measures $\mu_{B}$ converge weakly to a Gibbs measure on $\mathbf{Z}_{*}^{2}$. A natural question is whether or not there is a unique infinite-volume Gibbs measure. Nonuniqueness is equivalent to "a black boundary condition produces a mainly black pattern even in the limit" whereas uniqueness means that the boundary color is "forgotten in the limit".

It is well known (see e.g. [9]) that nonuniqueness in the Ising model is equivalent to percolation in the Fortuin-Kasteleyn random-cluster model with $q=2$ and $p_{e^{*}}=$ $1-\exp \left(-2 \ell_{e}\right)$ where $\ell_{e}$ is the length of the edge $e \in E$ crossing $e^{*}$. It is also well known that a random-cluster measure with parameters $q=2$ and $\left\{p_{e^{*}}, e^{*} \in E^{*}\right\}$ stochastically dominates Bernoulli percolation with parameters $\left\{p_{e^{*}}\left(p_{e^{*}}+2\left(1-p_{e^{*}}\right)\right)^{-1}\right\}$. In our case

$$
p_{e^{*}}\left(p_{e^{*}}+2\left(1-p_{e^{*}}\right)\right)^{-1}=\frac{1-\exp \left(-2 \ell_{e}\right)}{1+\exp \left(-2 \ell_{e}\right)} \geq 1-\exp \left(-\ell_{e}\right)
$$

and so by Proposition 3.4 nonuniqueness occurs for $\lambda<1$.

If Conjecture 3.2 holds, this would similarly imply uniqueness for $\lambda>2 \lambda_{c}$, and thereby the existence of a phase transition.

Remark. It was the above model that led us to consider stretched lattice. The black-and-white pattern model can in turn be seen a version of the Arak process for polygonal black-and-white patterns in $\mathbb{R}^{2}$ (see [1]). For this process G. Nicholls [13] uses a Peierls argument to prove phase coexistence, but the question of phase transition is wide open.

\section{Mixture of Bernoulli Percolations}

As in the previous example, we set $X_{e_{1}}=X_{e_{2}}$ for parallel $e_{1}, e_{2}$, and otherwise we set $X_{e_{1}}$ and $X_{e_{2}}$ to be independent. Here we take:

$$
X= \begin{cases}p_{1} & \text { with probability } 1-q \\ p_{2} & \text { with probability } q\end{cases}
$$


where $p_{1}<p_{c}\left(\mathbf{Z}^{d}\right)<p_{2}$. Again, it is easy to see that this model is monotone in $q$. Moreover, Theorem 1.1 implies:

Proposition 4.1 For $d \geq 3$, if

$$
(1-q) p_{1}^{-1}+q p_{2}^{-1}<\theta(d)^{-1}
$$

then a.s. there exists an oriented percolation cluster which is transient.

Proof: Again, we have condition (B) by definition, and

$$
\mathbf{E}\left[X^{-1}\right]=(1-q) p_{1}^{-1}+q p_{2}^{-1} .
$$

So condition (A) holds whenever (11) holds.

Proposition 4.1 together with Observation 4.2 below imply the existence of a phase transition for the existence of an oriented cluster when $p_{2}>\theta(d)$ and $p_{1}<d^{-1}$. We do not know how to prove such a phase transition for (unoriented) percolation, or for other values of $p_{1}, p_{2}$.

Observation 4.2 For all $d \geq 2$, if $p_{1}<d^{-1}$ and $q$ is small enough, there is a.s no infinite oriented percolation cluster.

Proof: Let $W_{n}$ be the number of open oriented paths of length $n$ which emanate from the origin. If we take $d\left((1-q) p_{1}+q p_{2}\right)<1$ we obtain:

$$
\mathbf{P}\left[W_{n} \geq 1\right] \leq \mathbf{E}\left[W_{n}\right]=d^{n}\left((1-q) p_{1}+q p_{2}\right)^{n} \rightarrow 0 .
$$

as $n \rightarrow \infty$. This implies that a.s., the origin is not in an infinite oriented cluster.

Remark: As noted by Geoffrey Grimmett (personal communication), similar problems were studied in the context of contact-processes (see, e.g, [4], [12], and [11]). However, in the models studied there the dependence is "one dimensional" while the dependence studied here is " $d$ dimensional". A different kind of dependent percolation was studied by Winkler [14] and by Balister, Bollobas and Stacey [2].

Acknowledgments: We are grateful to Itai Benjamini, Geoffrey Grimmett, Olle Häggström, and Geoff Nicholls for useful conversations. We thank Ariel Scolnicov for his help with figure 1.

\section{References}

[1] Arak, T., Clifford, P. and Surgailis, D. (1993). Point-based polygonal models for random graphs, Adv. Appl. Probab. 25, 348-372.

[2] Balister, P. N., Bollobas, B. and Stacey, A. M. (2000). Dependent percolation in two dimensions, Probab. Th. Rel. Fields, to appear.

[3] Benjamini, I., Pemantle, R. and Peres, Y. (1998). Unpredictable paths and percolation, Ann. Probab. 26, 1198-1211.

[4] Bramson, M., Durrett, R., and Schonmann, R. H. (1991). The contact process in a random environment Ann. Probab. 19, 960-983.

[5] T. Cox and R. Durrett (1983). Oriented percolation in dimensions $d \geq 4$ : bounds and asymptotic formulas. Math. Proc. Camb. Phil. Soc. 93, 151-162. 
[6] Doyle, P. G. and Snell, E. J. (1984). Random walks and electrical networks. Carus Math. Monographs 22, Math. Assoc. Amer., Washington, D. C.

[7] Grimmett, G. (1989). Percolation, Springer, New York.

[8] Grimmett, G. R., Kesten, H. and Zhang, Y. (1993). Random walk on the infinite cluster of the percolation model. Probab. Th. Rel. Fields 96, 33-44.

[9] Häggström, O. (1998). Random-cluster representations in the study of phase transitions, Markov Proc. Rel. Fields 4, 275-321.

[10] Häggström, O. and Mossel, E. (1998). Nearest-neighbor walks with low predictability profile and percolation in $2+\epsilon$ dimensions. Ann. Probab. 26, 12121231.

[11] Klein, A. (1994). Multiscale analysis in disordered systems: percolation and contact process in random environments, in Probability and Phase transition, Nato ASI series, Kluwer Academic Publishers, Dordecht.

[12] Liggett, T. (1992). The survival of one dimensional contact processes in random environments, Ann. Probab. 20, 696-723.

[13] Nicholls, G. (1996). Spontaneous magnetization in the plane, Technical report, University of Auckland.

[14] Winkler, P. (2000). Dependent percolation and colliding random walks, Rand. Struct. Alg. 16:1, 58-84. 